Erratum

\title{
Erratum: Ntana, F., et al. Aspergillus: A Powerful Protein Production Platform. Catalysts 2020, 10, 1064
}

\author{
Fani Ntana ${ }^{1}$, Uffe Hasbro Mortensen ${ }^{2}$, Catherine Sarazin ${ }^{1, *}$ and Rainer Figge ${ }^{1}$
}

1 Unité de Génie Enzymatique et Cellulaire, UMR 7025 CNRS/UPJV/UTC, Université de Picardie Jules Verne, 80039 Amiens, France; fani.ntana@u-picardie.fr (F.N.); rainer.figge@u-picardie.fr (R.F.)

2 Department of Biotechnology and Biomedicine, Technical University of Denmark, Søltofts Plads, Building 223, 2800 Kongens Lyngby, Denmark; um@bio.dtu.dk

* Correspondence: catherine.sarazin@u-picardie.fr; Tel.: +33-3-22-82-75-95

Received: 24 November 2020; Accepted: 25 November 2020; Published: 30 November 2020

The author wishes to make the following erratum to this paper [1]: Update due to some reporting errors in Tables 2, 8, 10 and 12.

Due to typographical errors concerning reference [47] and [51,52], replace:

Table 2. Approaches for improving recombinant protein production through promoter engineering.

\begin{tabular}{|c|c|c|c|c|}
\hline Process & Modification & Performance & $\begin{array}{l}\text { Improvement } \\
\text { Factor }\end{array}$ & Reference \\
\hline \multirow{4}{*}{ Promoters } & \multirow{4}{*}{$\begin{array}{l}\text { Use of several promoters } \\
\text { (P) in A. awamori }\end{array}$} & PB2 from Acremonium chrysogenum: $0.25-2 \mathrm{mg} / \mathrm{L}$ thaumatin & \multirow{4}{*}{-} & \multirow{4}{*}[46]{} \\
\hline & & PpcbC from Penicillium chrysogenum: $0.25-2 \mathrm{mg} / \mathrm{L}$ thaumatin & & \\
\hline & & PgdhA from A. awamori: $1-9 \mathrm{mg} / \mathrm{L}$ thaumatin & & \\
\hline & & PgpdA from A. nidulans: $0.75-11 \mathrm{mg} / \mathrm{L}$ thaumatin & & \\
\hline & $\begin{array}{l}\text { Insertion of multiple } \\
\text { copies of an activator } \\
\text { protein-binding site from } \\
\text { the cis-regulatory region } \\
\text { of } A \text {. niger gla } A \text { to the } \\
\text { new promoter in } A \text {. niger }\end{array}$ & $\begin{array}{c}396.0 \pm 51.5 \mathrm{mg} / \mathrm{L} \text { of Vitreoscilla hemoglobin compared to } \\
19.7 \pm 4.8 \mathrm{mg} / \mathrm{L} \text { from the strain with } 1 \text { copy }\end{array}$ & 20 & [45] \\
\hline & \multirow{3}{*}{$\begin{array}{l}\text { Use of hybrid promoters } \\
\text { (combination of a human } \\
\text { hERa-activated promoter } \\
\text { (pERE), S. cerevisiae } \\
\text { URA3 promoter and } \\
\text { A. nidulans nirA } \\
\text { promoter) in } A \text {. nidulans }\end{array}$} & $\begin{array}{c}\text { pERE-URA-nirA + lacZ: } 25 \mathrm{U} \text { of } \beta \text {-galactosidase activity/mg } \\
\text { of protein }\end{array}$ & - & \multirow{3}{*}{ [47] } \\
\hline & & $\begin{array}{c}\text { pERE-URA-RS (random stuffer-link) + lacZ: } 100 \mathrm{U} \text { of } \\
\beta \text {-galactosidase activity/mg of protein }\end{array}$ & 4 & \\
\hline & & $\begin{array}{c}\text { pERE-RS-nirA + lacZ: } 1400 \mathrm{U} \text { of } \beta \text {-galactosidase activity/mg } \\
\text { of protein } \\
{[1 \mathrm{pM} \text { inducer (DES) }]}\end{array}$ & 56 & \\
\hline & \multirow{3}{*}{$\begin{array}{l}\text { Use of a hemolysin-like } \\
\text { protein promoter (Phyl) } \\
\quad \text { for heterologous } \\
\text { production in A. oryzae }\end{array}$} & $\begin{array}{c}\text { Reporter gene: Endoglucanase Cel B } \\
\text { Pamy: } 24.1 \pm 5.5 \mathrm{U} / \mathrm{mL} \text {, Phyl: } 57.9 \pm 17.4 \mathrm{U} / \mathrm{mL}\end{array}$ & 2.4 & \multirow{3}{*}{ [48] } \\
\hline & & $\begin{array}{l}\text { Reporter gene: Trichoderma endoglucanase I } \\
\text { Pamy: } 7.7 \pm 3.9 \mathrm{U} / \mathrm{mL} \text {, Phyl: } 27.8 \pm 1.3 \mathrm{U} / \mathrm{mL}\end{array}$ & 3.6 & \\
\hline & & $\begin{array}{l}\text { Reporter gene: Trichoderma endoglucanase III } \\
\text { Pamy: } 4.0 \pm 0.6 \mathrm{U} / \mathrm{mL} \text {, hyl:31.7 } \pm 3.3 \mathrm{U} / \mathrm{mL}\end{array}$ & 7.9 & \\
\hline & \multirow{3}{*}{$\begin{array}{l}\text { Regulatory elements } \\
\text { (TerR and PterA) from } \\
\text { A. terreus terrain gene } \\
\text { cluster for E. coli lacZ } \\
\text { expression in A. niger }\end{array}$} & $\begin{array}{l}\text { Promoter activity } \sim 5000 \mathrm{mU} / \mathrm{mg} \text { when TerR under PgpdA } \\
\text { (No activity when TerR under the native promoter) }\end{array}$ & - & \multirow{3}{*}{ [49] } \\
\hline & & $\begin{array}{l}\text { Promoter activity } \sim 10,000 \mathrm{mU} / \mathrm{mg} \text { (when TerR under PgpdA } \\
\text { in } 2 \text { copies) }\end{array}$ & 2 & \\
\hline & & $\begin{array}{c}\text { Promoter activity } \sim 15,000 \mathrm{mU} / \mathrm{mg} \text { (when TerR under } \\
\text { PamyB) }\end{array}$ & 3 & \\
\hline
\end{tabular}


Table 2. Cont

\begin{tabular}{|c|c|c|c|c|c|}
\hline Process & \multicolumn{2}{|c|}{ Modification } & Performance & $\begin{array}{l}\text { Improvement } \\
\text { Factor }\end{array}$ & Reference \\
\hline \multicolumn{3}{|c|}{$\begin{array}{c}\text { A. niger } \\
\alpha \text {-glucosyltransferase } \\
\text { produced under the } \\
\text { A. niger pyruvate kinase } \\
\text { promoter }\end{array}$} & $\begin{array}{l}2000 \mathrm{U} / \mathrm{mL} \text { total activity of } \alpha \text {-glucosyltransferase compared } \\
\text { to } 600 \mathrm{U} / \mathrm{mL} \text { in the wild type }\end{array}$ & 3.3 & [50] \\
\hline \multicolumn{3}{|c|}{$\begin{array}{l}\text { Overexpression of the } \\
\text { transcription factor } \\
\text { RsmA, while the aflR } \\
\text { promoter was inserted in } \\
\text { front of the pslcc in } \\
\text { A. nidulans }\end{array}$} & $\begin{array}{c}\text { 60,000 U/mL of Pycnoporus sanguineus laccase compared to } \\
4000 \mathrm{U} / \mathrm{mL} \text { in the control strain }\end{array}$ & 15 & {$[51,52]$} \\
\hline \multicolumn{3}{|c|}{$\begin{array}{c}\text { A novel promoter from } \\
\text { Talaromyces emersonii } \\
\text { (Pglucan1200) for } \\
\text { expressing glaA in } \\
\text { A. niger }\end{array}$} & $\begin{array}{l}6000 \mathrm{U} / \mathrm{mL} \text { of GlaA, enzyme activity increased by about } 25 \% \\
\text { compared to } 5000 \mathrm{U} / \mathrm{mL} \text { in the strain with the PglaA }\end{array}$ & 1.2 & [53] \\
\hline \multirow{3}{*}{\multicolumn{2}{|c|}{$\begin{array}{l}\text { The } \\
\text { constitutive } \\
\text { promoter of } \\
\text { ecm33 } \\
\text { (Pecm33) } \\
\text { from A. niger } \\
\text { in A. niger }\end{array}$}} & Maltose: & $\begin{array}{c}\text { Pecm33 activity induced by } 1.7 \text { compared to PglaA activity } \\
\text { that induced by } 2.7\end{array}$ & \multirow{3}{*}{-} & \multirow{3}{*}{ [54] } \\
\hline & & Glucose: & $\begin{array}{c}\text { Pecm33 activity induced by } 1.1 \text { compared to PglaA activity } \\
\text { that induced by } 1.8\end{array}$ & & \\
\hline & & Xylose: & $\begin{array}{c}\text { Pecm33 activity induced by } 2 \text { compared to PglaA activity } \\
\text { that induced by } 1.3 \\
\text { Increased Pecm } 33 \text { activity at } 37^{\circ} \mathrm{C}\end{array}$ & & \\
\hline
\end{tabular}

with

Table 2. Approaches for improving recombinant protein production through promoter engineering.

\begin{tabular}{|c|c|c|c|c|}
\hline Process & Modification & Performance & $\begin{array}{l}\text { Improvement } \\
\text { Factor }\end{array}$ & Reference \\
\hline \multirow{3}{*}{ Promoters } & \multirow{3}{*}{$\begin{array}{l}\text { Use of several promoters } \\
\text { (P) in A. awamori }\end{array}$} & PB2 from Acremonium chrysogenum: $0.25-2 \mathrm{mg} / \mathrm{L}$ thaumatin & \multirow{3}{*}{-} & \multirow{3}{*}{ [46] } \\
\hline & & PgdhA from A. awamori: $1-9 \mathrm{mg} / \mathrm{L}$ thaumatin & & \\
\hline & & PgpdA from A. nidulans: $0.75-11 \mathrm{mg} / \mathrm{L}$ thaumatin & & \\
\hline & \multirow{2}{*}{$\begin{array}{l}\text { Use of hybrid promoters } \\
\text { (combination of a human } \\
\text { hERa-activated promoter } \\
\text { (pERE), S. cerevisiae } \\
\text { URA3 promoter and } \\
\text { A. nidulans nirA } \\
\text { promoter) in } A \text {. nidulans }\end{array}$} & $\begin{array}{c}\text { pERE-RS-nirA+ lacZ: } 25 \mathrm{U} \text { of } \beta \text {-galactosidase activity/mg of } \\
\text { protein }\end{array}$ & - & \multirow{2}{*}{ [47] } \\
\hline & & $\begin{array}{c}\text { pERE-URA-RS + lacZ: } 1400 \mathrm{U} \text { of } \beta \text {-galactosidase activity/mg } \\
\text { of protein } \\
{[1 \mathrm{pM} \text { inducer (DES) }]}\end{array}$ & 56 & \\
\hline & \multirow{3}{*}{$\begin{array}{l}\text { Use of a hemolysin-like } \\
\text { protein promoter (Phyl) } \\
\text { for heterologous } \\
\text { production in A. oryzae }\end{array}$} & $\begin{array}{c}\text { Reporter gene: Endoglucanase Cel B } \\
\text { Pamy: } 24.1 \pm 5.5 \mathrm{U} / \mathrm{mL} \text {, Phyl: } 57.9 \pm 17.4 \mathrm{U} / \mathrm{mL}\end{array}$ & 2.4 & \multirow{3}{*}{ [48] } \\
\hline & & $\begin{array}{l}\text { Reporter gene: Trichoderma endoglucanase I } \\
\text { Pamy: } 7.7 \pm 3.9 \mathrm{U} / \mathrm{mL} \text {, Phyl: } 27.8 \pm 1.3 \mathrm{U} / \mathrm{mL}\end{array}$ & 3.6 & \\
\hline & & $\begin{array}{l}\text { Reporter gene: Trichoderma endoglucanase III } \\
\text { Pamy:4.0 } \pm 0.6 \mathrm{U} / \mathrm{mL} \text {, hyl:31.7 } \pm 3.3 \mathrm{U} / \mathrm{mL}\end{array}$ & 7.9 & \\
\hline & $\begin{array}{l}\text { Regulatory elements } \\
\text { (TerR and PterA) from } \\
\text { A. terreus terrain gene } \\
\text { cluster for E. coli lacZ } \\
\text { expression in } A \text {. niger }\end{array}$ & $\begin{array}{l}\text { Promoter activity } \sim 5000 \mathrm{mU} / \mathrm{mg} \text { when TerR under PgpdA } \\
\text { (No activity when TerR under the native promoter) }\end{array}$ & - & [49] \\
\hline
\end{tabular}


Table 2. Cont.

\begin{tabular}{|c|c|c|c|c|c|}
\hline Process & \multicolumn{2}{|c|}{ Modification } & Performance & $\begin{array}{l}\text { Improvement } \\
\text { Factor }\end{array}$ & Reference \\
\hline \multicolumn{3}{|c|}{$\begin{array}{c}\text { A. niger } \\
\alpha \text {-glucosyltransferase } \\
\text { produced under the } \\
\text { A. niger pyruvate } \\
\text { kinase promoter }\end{array}$} & $\begin{array}{l}2000 \mathrm{U} / \mathrm{mL} \text { total activity of } \alpha \text {-glucosyltransferase compared } \\
\text { to } 600 \mathrm{U} / \mathrm{mL} \text { in the wild type }\end{array}$ & 3.3 & [50] \\
\hline \multicolumn{3}{|c|}{$\begin{array}{c}\text { A novel promoter from } \\
\text { Talaromyces emersonii } \\
\text { (Pglucan1200) for } \\
\text { expressing glaA in } \\
\text { A. niger }\end{array}$} & $\begin{array}{l}6000 \mathrm{U} / \mathrm{mL} \text { of GlaA compared to } 5000 \mathrm{U} / \mathrm{mL} \text { in the strain } \\
\text { with the PglaA }\end{array}$ & 1.2 & [53] \\
\hline \multirow{3}{*}{\multicolumn{2}{|c|}{$\begin{array}{c}\text { The } \\
\text { constitutive } \\
\text { promoter of } \\
\text { ecm33 } \\
\text { (Pecm33) } \\
\text { from A. niger } \\
\text { in A. niger }\end{array}$}} & Maltose: & $\begin{array}{c}\text { Pecm33 activity induced by } 1.7 \text { compared to PglaA activity } \\
\text { that induced by } 2.7\end{array}$ & \multirow{3}{*}{-} & \multirow{3}{*}{ [54] } \\
\hline & & Glucose: & $\begin{array}{c}\text { Pecm33 activity induced by } 1.1 \text { compared to PglaA activity } \\
\text { that induced by } 1.8\end{array}$ & & \\
\hline & & Xylose: & $\begin{array}{c}\text { Pecm33 activity induced by } 2 \text { compared to PglaA activity } \\
\text { that induced by } 1.3 \\
\text { Increased Pecm } 33 \text { activity at } 37^{\circ} \mathrm{C}\end{array}$ & & \\
\hline
\end{tabular}

Due to a typographical error concerning reference [109], replace:

Table 8. Approaches for improving recombinant protein production through engineering protein degradation pathways.

\begin{tabular}{|c|c|c|c|c|}
\hline Process & Modification & Performance & $\begin{array}{l}\text { Improvement } \\
\text { Factor }\end{array}$ & Reference \\
\hline \multirow{7}{*}{$\begin{array}{l}\text { Protein degradation } \\
\text { pathways- ERAD } \\
\text { and Vacuole }\end{array}$} & $\begin{array}{l}\text { Deletion of } \operatorname{der} A \\
\text { and } \operatorname{der} B \text { in } A \text {. niger }\end{array}$ & $\begin{array}{c}\Delta \text { derA: } 80 \% \text { decrease in Tramete laccase } \\
\text { production }\end{array}$ & 0.2 & [99] \\
\hline & - & $\Delta$ derB: $15.7 \%$ increase in Tramete laccase & 1.15 & \\
\hline & $\begin{array}{l}\text { Deletion of doaA } \\
\text { and overexpression } \\
\text { of } s t t C \text { in } A \text {. niger }\end{array}$ & $\begin{array}{l}\text { Higher GUS activity compared to } \\
\text { parental strain (no quantitative } \\
\text { data available) }\end{array}$ & - & [106] \\
\hline & \multirow{2}{*}{$\begin{array}{c}\text { Disruption of } \\
\text { Aovps10 in A. oryzae }\end{array}$} & $\begin{array}{l}83.1 \text { and } 70.3 \mathrm{mg} / \mathrm{L} \text { chymosin compared } \\
\text { to } 28.7 \mathrm{mg} / \mathrm{L} \text { in parental strain }\end{array}$ & $3-2.5$ & \multirow{2}{*}{ [108] } \\
\hline & & $\begin{array}{l}22.6 \text { and } 24.6 \mathrm{mg} / \mathrm{L} \text { human lysozyme } \\
\text { compared to } 11.1 \mathrm{mg} / \mathrm{L} \text { in parental strain }\end{array}$ & $2-2.2$ & \\
\hline & \multirow[b]{2}{*}{$\begin{array}{l}\text { Deletion of ERAD } \\
\text { key genes }(\text { der } A \text {, } \\
\text { doaA, } h r d C, \text { mifA } \\
\text { and } m n s A) \text { in } \\
\text { A. niger }\end{array}$} & $\begin{array}{c}\Delta \text { derA and } \Delta \text { hrdC: } 2 \text {-fold increase } \\
\text { compared to parental strain (single-copy) }\end{array}$ & 2 & \multirow[b]{2}{*}{ [107] } \\
\hline & & $\begin{array}{l}\Delta \text { derA: 6-fold increase compared to } \\
\text { parental strain (multi-copy) } \\
\text { Relative amount of intracellular GlaGus } \\
\text { ( } \beta \text {-glucuronidase levels) fusion protein } \\
\text { detected in total protein extracts of strains } \\
\text { with impaired ERAD and respective } \\
\text { parental strain }\end{array}$ & 6 & \\
\hline
\end{tabular}


Table 8. Cont.

\begin{tabular}{|c|c|c|c|c|}
\hline Process & Modification & Performance & $\begin{array}{c}\text { Improvement } \\
\text { Factor }\end{array}$ & Reference \\
\hline & \multirow{6}{*}{$\begin{array}{l}\text { Disruption of } \\
\text { genes involved in } \\
\text { autophagy in } \\
\text { A. oryzae }\end{array}$} & $\Delta$ Aoatg1: $60 \mathrm{mg} / \mathrm{L}$ chymosin & 2.3 & \multirow{6}{*}{ [109] } \\
\hline & & $\Delta$ Aoatg13: $37 \mathrm{mg} / \mathrm{L}$ chymosin & 1.4 & \\
\hline & & $\Delta$ Aoatg $4: 80 \mathrm{mg} / \mathrm{L}$ chymosin & 3.1 & \\
\hline & & $\Delta$ Aoatg8: $66 \mathrm{mg} / \mathrm{L}$ chymosin & 2.5 & \\
\hline & & $\Delta$ Aoatg15: Not detectable & - & \\
\hline & & Control: $26 \mathrm{mg} / \mathrm{L}$ chymosin & - & \\
\hline
\end{tabular}

with

Table 8. Approaches for improving recombinant protein production through engineering protein degradation pathways.

\begin{tabular}{|c|c|c|c|c|}
\hline Process & Modification & Performance & $\begin{array}{l}\text { Improvement } \\
\text { Factor }\end{array}$ & Reference \\
\hline \multirow{13}{*}{$\begin{array}{l}\text { Protein degradation } \\
\text { pathways-ERAD } \\
\text { and Vacuole }\end{array}$} & $\begin{array}{l}\text { Deletion of } \operatorname{der} A \\
\text { and } \operatorname{der} B \text { in } A \text {. niger }\end{array}$ & $\begin{array}{c}\Delta \text { derA: } 80 \% \text { decrease in Tramete laccase } \\
\text { production }\end{array}$ & 0.2 & \multirow[t]{2}{*}{ [99] } \\
\hline & - & $\Delta$ derB: $15.7 \%$ increase in Tramete laccase & 1.15 & \\
\hline & $\begin{array}{l}\text { Deletion of } d o a A \\
\text { and overexpression } \\
\text { of } s t t C \text { in } A \text {. niger }\end{array}$ & $\begin{array}{c}\text { Higher GUS activity compared to } \\
\text { parental strain (no quantitative data } \\
\text { available) }\end{array}$ & - & [106] \\
\hline & \multirow{2}{*}{$\begin{array}{c}\text { Disruption of } \\
\text { Aovps10 in A. oryzae }\end{array}$} & $\begin{array}{l}83.1 \text { and } 70.3 \mathrm{mg} / \mathrm{L} \text { chymosin compared } \\
\text { to } 28.7 \mathrm{mg} / \mathrm{L} \text { in parental strain }\end{array}$ & $3-2.5$ & \multirow{2}{*}{ [108] } \\
\hline & & $\begin{array}{l}22.6 \text { and } 24.6 \mathrm{mg} / \mathrm{L} \text { human lysozyme } \\
\text { compared to } 11.1 \mathrm{mg} / \mathrm{L} \text { in parental strain }\end{array}$ & $2-2.2$ & \\
\hline & \multirow[b]{2}{*}{$\begin{array}{l}\text { Deletion of ERAD } \\
\text { key genes (der } A \text {, } \\
\text { doaA, } h r d C, \text { mifA } \\
\text { and } m n s A) \text { in } \\
\text { A. niger }\end{array}$} & $\begin{array}{l}\Delta \text { derA and } \Delta \text { hrdC: } 2 \text {-fold increase } \\
\text { compared to parental strain (single-copy) }\end{array}$ & 2 & \multirow[b]{2}{*}{ [107] } \\
\hline & & $\begin{array}{l}\Delta \text { derA: 6-fold increase compared to } \\
\text { parental strain (multi-copy) } \\
\text { Relative amount of intracellular GlaGus } \\
\text { ( } \beta \text {-glucuronidase levels) fusion protein } \\
\text { detected in total protein extracts of strains } \\
\text { with impaired ERAD and respective } \\
\text { parental strain }\end{array}$ & 6 & \\
\hline & \multirow{6}{*}{$\begin{array}{l}\text { Disruption of } \\
\text { genes involved in } \\
\text { autophagy in } \\
\text { A. oryzae }\end{array}$} & $\Delta$ Aoatg1: $60 \mathrm{mg} / \mathrm{L}$ chymosin & 2.3 & \multirow{6}{*}{ [109] } \\
\hline & & $\Delta$ Aoatg13: $37 \mathrm{mg} / \mathrm{L}$ chymosin & 1.4 & \\
\hline & & $\Delta$ Aoatg4: $80 \mathrm{mg} / \mathrm{L}$ chymosin & 3.1 & \\
\hline & & $\Delta$ Aoatg8: $66 \mathrm{mg} / \mathrm{L}$ chymosin & 2.5 & \\
\hline & & $\Delta$ Aoatg15: $24 \mathrm{mg} / \mathrm{L}$ chymosin & 1 & \\
\hline & & Control: $26 \mathrm{mg} / \mathrm{L}$ chymosin & - & \\
\hline
\end{tabular}

Due to typographical errors concerning reference [126] and [51], replace: 
Table 10. Approaches for improving recombinant protein production through disruption of protease genes.

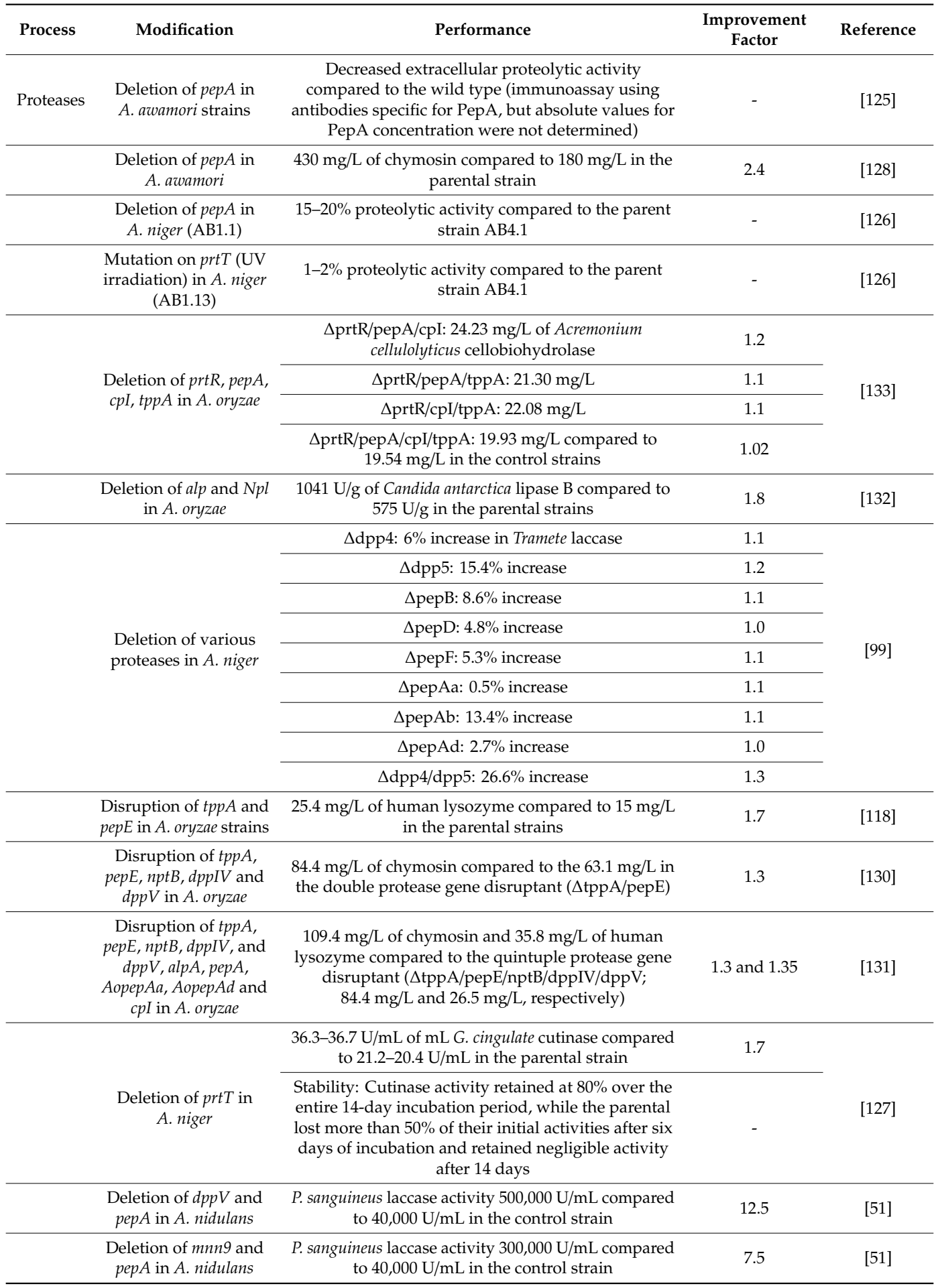

with 
Table 10. Approaches for improving recombinant protein production through disruption of protease genes.

\begin{tabular}{|c|c|c|c|c|}
\hline Process & Modification & Performance & $\begin{array}{l}\text { Improvement } \\
\text { Factor }\end{array}$ & Reference \\
\hline \multirow[t]{8}{*}{ Proteases } & $\begin{array}{l}\text { Deletion of pepA in } \\
\text { A. awamori strains }\end{array}$ & $\begin{array}{l}\text { Decreased extracellular proteolytic activity } \\
\text { compared to the wild type (immunoassay using } \\
\text { antibodies specific for PepA, but absolute values for } \\
\text { PepA concentration were not determined) }\end{array}$ & - & [125] \\
\hline & $\begin{array}{l}\text { Deletion of pepA in } \\
\text { A. awamori }\end{array}$ & $\begin{array}{l}430 \mathrm{mg} / \mathrm{L} \text { of chymosin compared to } 180 \mathrm{mg} / \mathrm{L} \text { in the } \\
\text { parental strain }\end{array}$ & 2.4 & [128] \\
\hline & $\begin{array}{l}\text { Deletion of pepA in } \\
\text { A. niger (AB1.18) }\end{array}$ & $\begin{array}{l}15-20 \% \text { proteolytic activity compared to the parent } \\
\text { strain AB4.1 }\end{array}$ & - & [126] \\
\hline & $\begin{array}{l}\text { Mutation on } p r t T(\mathrm{UV} \\
\text { irradiation) in } A \text {. niger } \\
\text { (AB1.13) }\end{array}$ & $\begin{array}{l}1-2 \% \text { proteolytic activity compared to the parent } \\
\text { strain AB4.1 }\end{array}$ & - & [126] \\
\hline & \multirow{4}{*}{$\begin{array}{l}\text { Deletion of } p r t R, \text { pepA, } \\
c p I, t p p A \text { in } A \text {. oryzae }\end{array}$} & $\begin{array}{l}\Delta \text { prtR/pepA/cpI: } 24.23 \mathrm{mg} / \mathrm{L} \text { of Acremonium } \\
\text { cellulolyticus cellobiohydrolase }\end{array}$ & 1.2 & \multirow{4}{*}{ [133] } \\
\hline & & $\Delta$ prtR/pepA/tppA: $21.30 \mathrm{mg} / \mathrm{L}$ & 1.1 & \\
\hline & & $\Delta$ prtR/cpI/tppA: $22.08 \mathrm{mg} / \mathrm{L}$ & 1.1 & \\
\hline & & $\begin{array}{c}\Delta \text { prtR/pepA/cpI/tppA: } 19.93 \mathrm{mg} / \mathrm{L} \text { compared to } \\
19.54 \mathrm{mg} / \mathrm{L} \text { in the control strains }\end{array}$ & 1.02 & \\
\hline & $\begin{array}{l}\text { Deletion of alp and } \mathrm{Npl} \\
\quad \text { in A. oryzae }\end{array}$ & $\begin{array}{c}1041 \mathrm{U} / \mathrm{g} \text { of Candida antarctica lipase B compared to } \\
575 \mathrm{U} / \mathrm{g} \text { in the parental strains }\end{array}$ & 1.8 & [132] \\
\hline & \multirow{9}{*}{$\begin{array}{l}\text { Deletion of various } \\
\text { proteases in } A \text {. niger }\end{array}$} & $\Delta$ dpp $4: 6 \%$ increase in Tramete laccase & 1.1 & \multirow{9}{*}[99]{} \\
\hline & & $\Delta$ dpp5: $15.4 \%$ increase & 1.2 & \\
\hline & & $\Delta$ pepB: $8.6 \%$ increase & 1.1 & \\
\hline & & $\Delta$ pepD: $4.8 \%$ increase & 1.0 & \\
\hline & & $\Delta$ pepF: $5.3 \%$ increase & 1.1 & \\
\hline & & $\Delta$ pepAa: $0.5 \%$ increase & 1.1 & \\
\hline & & $\Delta$ pepAb: $13.4 \%$ increase & 1.1 & \\
\hline & & $\Delta$ pepAd: $2.7 \%$ increase & 1.0 & \\
\hline & & $\Delta \mathrm{dpp} 4 / \mathrm{dpp} 5: 26.6 \%$ increase & 1.3 & \\
\hline & $\begin{array}{l}\text { Disruption of } t p p A \text { and } \\
\text { pepE in } A \text {. oryzae strains }\end{array}$ & $\begin{array}{c}25.4 \mathrm{mg} / \mathrm{L} \text { of human lysozyme compared to } 15 \mathrm{mg} / \mathrm{L} \\
\text { in the parental strains }\end{array}$ & 1.7 & [118] \\
\hline & $\begin{array}{l}\text { Disruption of } t p p A, \\
p e p E, n p t B, d p p I V \text { and } \\
d p p V \text { in } A \text {. oryzae }\end{array}$ & $\begin{array}{l}84.4 \mathrm{mg} / \mathrm{L} \text { of chymosin compared to the } 63.1 \mathrm{mg} / \mathrm{L} \text { in } \\
\text { the double protease gene disruptant }(\Delta \mathrm{tppA} / \mathrm{pepE})\end{array}$ & 1.3 & [130] \\
\hline & $\begin{array}{l}\text { Disruption of } t p p A, \\
\text { pepE, } n p t B, \operatorname{dpp} I V \text {, and } \\
\operatorname{dpp} V, \operatorname{alp} A, p e p A, \\
\text { AopepAa, AopepAd and } \\
\text { cpI in A. oryzae }\end{array}$ & $\begin{array}{l}109.4 \mathrm{mg} / \mathrm{L} \text { of chymosin and } 35.8 \mathrm{mg} / \mathrm{L} \text { of human } \\
\text { lysozyme compared to the quintuple protease gene } \\
\text { disruptant }(\Delta \operatorname{tpp} \mathrm{A} / \mathrm{pepE} / \mathrm{nptB} / \mathrm{dppIV} / \mathrm{dppV} \\
84.4 \mathrm{mg} / \mathrm{L} \text { and } 26.5 \mathrm{mg} / \mathrm{L} \text {, respectively) }\end{array}$ & 1.3 and 1.35 & [131] \\
\hline \multirow{2}{*}{\multicolumn{2}{|c|}{$\begin{array}{l}\text { Deletion of prtT in } \\
\quad \text { A. niger }\end{array}$}} & $\begin{array}{l}36.3-36.7 \mathrm{U} / \mathrm{mL} \text { of } \mathrm{mL} \mathrm{G} \text {. cingulate cutinase compared } \\
\text { to } 21.2-20.4 \mathrm{U} / \mathrm{mL} \text { in the parental strain }\end{array}$ & 1.7 & \multirow[b]{2}{*}[127]{} \\
\hline & & $\begin{array}{l}\text { Stability: Cutinase activity retained at } 80 \% \text { over the } \\
\text { entire } 14 \text {-day incubation period, while the parental } \\
\text { lost more than } 50 \% \text { of their initial activities after six } \\
\text { days of incubation and retained negligible activity } \\
\text { after } 14 \text { days }\end{array}$ & - & \\
\hline & $\begin{array}{l}\text { Deletion of } d p p V \text { and } \\
\text { pepA in } A \text {. nidulans }\end{array}$ & $\begin{array}{l}\text { P. sanguineus laccase activity } 0.5 \mathrm{U} / \mathrm{mL} \text { compared to } \\
0.04 \mathrm{U} / \mathrm{mL} \text { in the control strain }\end{array}$ & 12.5 & [51] \\
\hline & $\begin{array}{l}\text { Deletion of mnn9 and } \\
\text { pepA in A. nidulans }\end{array}$ & $\begin{array}{l}\text { P. sanguineus laccase activity } 0.3 \mathrm{U} / \mathrm{mL} \text { compared to } \\
0.04 \mathrm{U} / \mathrm{mL} \text { in the control strain }\end{array}$ & 7.5 & {$[51]$} \\
\hline
\end{tabular}

Due to a typographical error concerning reference [144], replace: 
Table 12. Approaches for improving recombinant protein production through bioprocessing modifications.

\begin{tabular}{|c|c|c|c|c|}
\hline Process & Modification & Performance & $\begin{array}{l}\text { Improvement } \\
\text { Factor }\end{array}$ & Reference \\
\hline \multirow{10}{*}{$\begin{array}{l}\text { Fermentation } \\
\text { conditions }\end{array}$} & \multirow{6}{*}{$\begin{array}{l}\text { Effect of growth medium and } \\
\text { temperature on hen egg white lysozyme } \\
\text { (HEWL) production in } A \text {. niger }\end{array}$} & $\begin{array}{l}20-25^{\circ} \mathrm{C} 8-10 \mathrm{mg} / \mathrm{L} \\
\text { HEWL while } 30-37^{\circ} \mathrm{C} \\
\text { 3-5 mg/L HEWL }\end{array}$ & $\begin{array}{l}\text { Temperature: } \\
2-2.6\end{array}$ & \multirow{6}{*}[141]{} \\
\hline & & $\begin{array}{c}\text { soluble starch: } 8.0 \mathrm{mg} / \mathrm{L} \\
\text { HEWL }\end{array}$ & $\begin{array}{c}\text { Carbon source: } \\
1.7-2\end{array}$ & \\
\hline & & maltose: $4.5 \mathrm{mg} / \mathrm{L}$ HEWL & - & \\
\hline & & glucose: $4.0 \mathrm{mg} / \mathrm{L} \mathrm{HEWL}$ & - & \\
\hline & & xylose: $0.2 \mathrm{mg} / \mathrm{L} \mathrm{HEWL}$ & - & \\
\hline & & $\begin{array}{l}\text { soy milk medium: } \\
\text { 30-60 mg/L HEWL }\end{array}$ & $\begin{array}{l}\text { Rich medium: } \\
\quad 3.8-7.5\end{array}$ & \\
\hline & \multirow{4}{*}{$\begin{array}{l}\text { Effect of organic nitrogen sources on } \\
\text { recombinant glucoamylase production in } \\
\text { A. niger }\end{array}$} & $\begin{array}{l}\text { Unsupplemented: } 44 \mathrm{mg} \\
\text { glucoamylase/g biomass }\end{array}$ & - & \multirow{4}{*}[143]{} \\
\hline & & $\begin{array}{c}\text { L-alanine: } 32 \mathrm{mg} \\
\text { glucoamylase/g biomass }\end{array}$ & 0.7 & \\
\hline & & $\begin{array}{l}\text { L-methionine: } 26 \mathrm{mg} \\
\text { glucoamylase/g }\end{array}$ & 0.6 & \\
\hline & & $\begin{array}{l}\text { casamino acids, yeast } \\
\text { extract, peptone, and } \\
\text { gelatin: } 100 \mathrm{mg} \\
\text { glucoamylase/g }\end{array}$ & 2.2 & \\
\hline & \multirow{3}{*}{$\begin{array}{l}\text { Effect of agitation intensity on } \\
\text { recombinant amyloglucosidase (AMG) } \\
\text { production in A. oryzae }\end{array}$} & $\begin{array}{l}\text { Titer at the end of the } \\
\text { batch phase } \\
525 \text { rpm: } 110 \text { U/L AMG }\end{array}$ & - & \multirow{3}{*}{ [146] } \\
\hline & & 675 rpm: $230 \mathrm{U} / \mathrm{L} \mathrm{AMG}$ & 1.6 & \\
\hline & & 825 rpm: $370 \mathrm{U} / \mathrm{L}$ AMG & 3.3 & \\
\hline & $\begin{array}{c}\text { Effects of bioprocess } \\
\text { parameters-agitation intensity, initial } \\
\text { glucose concentration, initial yeast extract } \\
\text { concentration, and dissolved oxygen } \\
\text { tension (DO) - on heterologous protein } \\
\text { production in A. oryzae }\end{array}$ & $\begin{array}{l}\text { Highest GFP yields were } \\
\text { achieved under these } \\
\text { conditions: agitation } 400 \\
\text { rpm, glucose } 25 \mathrm{~g} / \mathrm{L} \text {, yeast } \\
\text { extract } 0 \mathrm{~g} / \mathrm{dm}^{3} \text {, DO } 15 \%\end{array}$ & - & [142] \\
\hline & \multirow{3}{*}{$\begin{array}{l}\text { Effect of agitation intensity on } \\
\text { recombinant glucose oxidase production } \\
\text { in } A \text {. niger }\end{array}$} & $\begin{array}{l}200 \mathrm{rpm}: 300 \mathrm{mkat} / \mathrm{L} \text { of } \\
\text { glucose oxidase }\end{array}$ & - & \multirow{3}{*}[144]{} \\
\hline & & $\begin{array}{l}500 \mathrm{rpm}: 800 \mathrm{mkat} / \mathrm{L} \text { of } \\
\text { glucose oxidase }\end{array}$ & 2.6 & \\
\hline & & $\begin{array}{l}800 \mathrm{rpm}: 600 \mathrm{mkat} / \mathrm{L} \text { of } \\
\text { glucose oxidase }\end{array}$ & 1.3 & \\
\hline & \multirow{5}{*}{$\begin{array}{c}\text { Effect of temperature on Pleurotus eryngii } \\
\text { versatile peroxidase production in } \\
\text { A. nidulans and A. niger }\end{array}$} & $\begin{array}{c}-A . \text { nidulans } \\
31^{\circ} \mathrm{C}: 24 \mathrm{U} / \mathrm{L} \text { peroxidase } \\
\text { activity }\end{array}$ & - & \multirow{5}{*}{ [145] } \\
\hline & & $\begin{array}{c}28^{\circ} \mathrm{C}: 80 \mathrm{U} / \mathrm{L} \text { peroxidase } \\
\text { activity }\end{array}$ & 3.3 & \\
\hline & & $\begin{array}{c}19^{\circ} \mathrm{C}: 466 \mathrm{U} / \mathrm{L} \text { peroxidase } \\
\text { activity }\end{array}$ & 19.4 & \\
\hline & & $\begin{array}{c}-A \text {. niger } \\
28^{\circ} \mathrm{C}: 107 \mathrm{U} / \mathrm{L} \text { peroxidase } \\
\text { activity }\end{array}$ & - & \\
\hline & & $\begin{array}{c}19^{\circ} \mathrm{C}: 412 \mathrm{U} / \mathrm{L} \text { peroxidase } \\
\text { activity }\end{array}$ & 3.8 & \\
\hline \multirow{2}{*}{$\begin{array}{l}\text { Fungal } \\
\text { morphology }\end{array}$} & \multirow{2}{*}{$\begin{array}{l}\text { Effect of raising the viscosity of the } \\
\text { medium by addition of } \\
\text { polyvinylpyrrolidone-PVP (transition } \\
\text { from aggregated mycelia (pellets) to } \\
\text { dispersed mycelia) on hen egg white } \\
\text { lysozyme (HEWL) in } A \text {. niger }\end{array}$} & $\begin{array}{l}\text { Medium with no PVP: } \\
110 \mathrm{mg} / \mathrm{L} \text { fresh and } 8 \mathrm{mg} / \mathrm{g} \\
\text { dry weight of HEWL }\end{array}$ & \multirow{2}{*}{1.7} & \multirow{2}{*}{ [147] } \\
\hline & & $\begin{array}{c}\text { Medium with PVP: } \\
190 \mathrm{mg} / \mathrm{L} \text { fresh and } \\
14 \mathrm{mg} / \mathrm{g} \text { dry weight of } \\
\text { HEWL }\end{array}$ & & \\
\hline
\end{tabular}


Table 12. Cont.

\begin{tabular}{|c|c|c|c|c|}
\hline Process & Modification & Performance & $\begin{array}{l}\text { Improvement } \\
\text { Factor }\end{array}$ & Reference \\
\hline & \multirow[b]{2}{*}{$\begin{array}{l}\text { Effect of addition of microparticles } \\
\text { (linked to the formation of freely } \\
\text { dispersed mycelium) on titers of native } \\
\text { glucoamylase (GlaA) and recombinant } \\
\text { fructofuranosidase (FF) produced in } \\
\text { A. niger }\end{array}$} & $\begin{array}{l}\text { No microparticles: } \\
17 \mathrm{U} / \mathrm{mL} \text { GlaA and } \\
42 \mathrm{U} / \mathrm{mL} \mathrm{FF}\end{array}$ & & \multirow[b]{2}{*}{ [148] } \\
\hline & & $\begin{array}{c}\text { Talc microparticles: } \\
61 \mathrm{U} / \mathrm{mL} \text { GlaA and } \\
92 \mathrm{U} / \mathrm{mL} \text { FF } \\
\text { FF production can reach } \\
\text { up to } 160 \mathrm{U} / \mathrm{mL}(10 \mathrm{~g} / \mathrm{L} \text { talc } \\
\text { microparticles of size } \\
6 \mathrm{~mm})\end{array}$ & $\begin{array}{l}3.5 \mathrm{GlaA} \\
2-3.8 \mathrm{FF}\end{array}$ & \\
\hline & \multirow{2}{*}{$\begin{array}{l}\text { Effect of addition of titanate } \\
\text { microparticles ( } \mathrm{TiSiO}_{4}, 8 \mathrm{~mm} \text { ) on titers of } \\
\text { native glucoamylase }(\mathrm{GlaA}) \text { and } \\
\text { recombinant fructofuranosidase }(\mathrm{FF}) \\
\text { produced in } A \text {. niger }\end{array}$} & $\begin{array}{l}\text { No microparticles: } \\
19 \text { U/mL GlaA and } \\
40 \mathrm{U} / \mathrm{mL} \text { FF }\end{array}$ & \multirow{2}{*}{$\begin{array}{l}\text { 9.5 GlaA } \\
3.7 \mathrm{FF}\end{array}$} & \multirow{2}{*}{ [149] } \\
\hline & & $\begin{array}{l}\text { Microparticles: } 190 \mathrm{U} / \mathrm{mL} \\
\text { glucoamylase and } \\
150 \mathrm{U} / \mathrm{mL} \\
\text { fructofuranosidase }\end{array}$ & & \\
\hline & \multirow{3}{*}{$\begin{array}{c}\text { Effect of growth type on hen egg white } \\
\text { lysozyme (HEWL) production and } \\
\text { protease activity in } A \text {. niger }\end{array}$} & $\begin{array}{c}\text { Free suspension: } \\
5.8 \mathrm{mg} / \mathrm{g} \text { HEWL } \\
95.3 \mathrm{U} / \mathrm{g} \text { Protease activity }\end{array}$ & 1.5 & \multirow{3}{*}{ [140] } \\
\hline & & $\begin{array}{c}\text { Mycelial pellets: } \\
5.0 \mathrm{mg} / \mathrm{g} \text { HEWL } \\
58.6 \mathrm{U} / \mathrm{g} \text { Protease activity }\end{array}$ & 1.2 & \\
\hline & & $\begin{array}{c}\text { Celite-560-immobilized } \\
\text { cultures: } \\
4.1 \mathrm{mg} / \mathrm{g} \text { HEWL } \\
56.3 \mathrm{U} / \mathrm{g} \text { Protease activity }\end{array}$ & - & \\
\hline
\end{tabular}

with

Table 12. Approaches for improving recombinant protein production through bioprocessing modifications.

\begin{tabular}{|c|c|c|c|c|}
\hline Process & Modification & Performance & $\begin{array}{l}\text { Improvement } \\
\text { Factor }\end{array}$ & Reference \\
\hline \multirow{6}{*}{$\begin{array}{l}\text { Fermentation } \\
\text { conditions }\end{array}$} & \multirow{6}{*}{$\begin{array}{l}\text { Effect of growth medium and } \\
\text { temperature on hen egg white lysozyme } \\
\text { (HEWL) production in } A \text {. niger }\end{array}$} & $\begin{array}{l}20-25^{\circ} \mathrm{C} 8-10 \mathrm{mg} / \mathrm{L} \\
\text { HEWL while } 30-37^{\circ} \mathrm{C} \\
\text { 3-5 mg/L HEWL }\end{array}$ & $\begin{array}{l}\text { Temperature: } \\
2-2.6\end{array}$ & \multirow{6}{*}{ [141] } \\
\hline & & $\begin{array}{c}\text { soluble starch: } 8.0 \mathrm{mg} / \mathrm{L} \\
\text { HEWL }\end{array}$ & $\begin{array}{c}\text { Carbon source: } \\
1.7-2\end{array}$ & \\
\hline & & maltose: $4.5 \mathrm{mg} / \mathrm{L}$ HEWL & - & \\
\hline & & glucose: $4.0 \mathrm{mg} / \mathrm{L}$ HEWL & - & \\
\hline & & xylose:0.2 mg/L HEWL & - & \\
\hline & & $\begin{array}{l}\text { soy milk medium: } \\
30-60 \mathrm{mg} / \mathrm{L} \text { HEWL }\end{array}$ & $\begin{array}{l}\text { Rich medium: } \\
3.8-7.5\end{array}$ & \\
\hline & \multirow{4}{*}{$\begin{array}{c}\text { Effect of organic nitrogen sources on } \\
\text { recombinant glucoamylase production in } \\
\text { A. niger }\end{array}$} & $\begin{array}{l}\text { Unsupplemented: } 44 \mathrm{mg} \\
\text { glucoamylase/g biomass }\end{array}$ & - & \multirow{4}{*}{ [143] } \\
\hline & & $\begin{array}{c}\text { L-alanine: } 32 \mathrm{mg} \\
\text { glucoamylase/g biomass }\end{array}$ & 0.7 & \\
\hline & & $\begin{array}{l}\text { L-methionine: } 26 \mathrm{mg} \\
\text { glucoamylase/g }\end{array}$ & 0.6 & \\
\hline & & $\begin{array}{l}\text { casamino acids, yeast } \\
\text { extract, peptone, and } \\
\text { gelatin: } 100 \mathrm{mg} \\
\text { glucoamylase } / \mathrm{g}\end{array}$ & 2.2 & \\
\hline
\end{tabular}


Table 12. Cont.

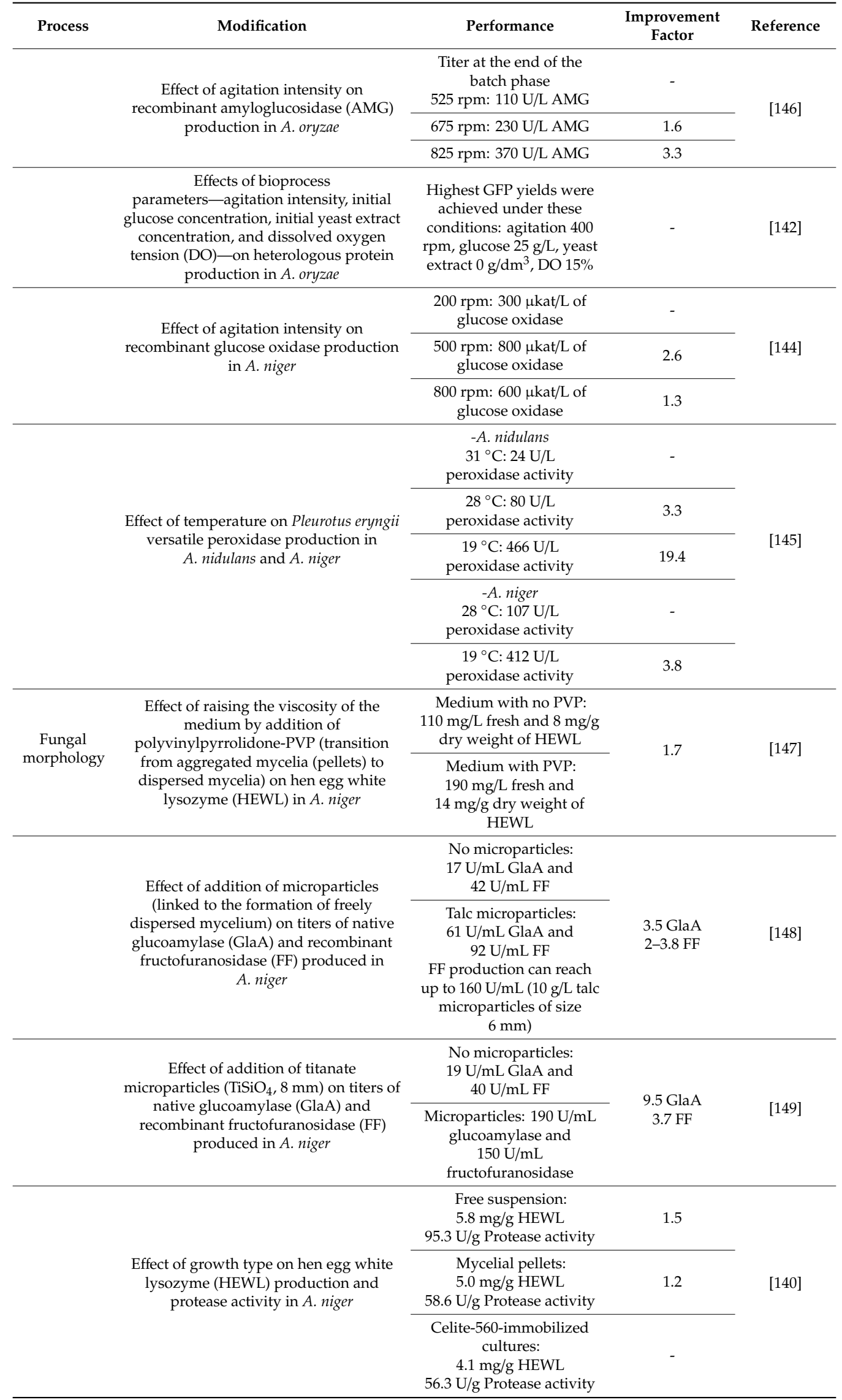


This update does not change any of the scientific results of the paper. The authors would like to apologize for any inconvenience caused to the readers by these changes. The manuscript will be updated and the original will remain online on the article webpage: https:/www.mdpi.com/2073-4344/10/9/1064 .

\section{Reference}

1. Ntana, F.; Mortensen, U.H.; Sarazin, C.; Figge, R. Aspergillus: A Powerful Protein Production Platform. Catalysts 2020, 10, 1064. [CrossRef]

Publisher's Note: MDPI stays neutral with regard to jurisdictional claims in published maps and institutional affiliations.

(C) 2020 by the authors. Licensee MDPI, Basel, Switzerland. This article is an open access article distributed under the terms and conditions of the Creative Commons Attribution (CC BY) license (http://creativecommons.org/licenses/by/4.0/). 